ten gefärbten und ungefärbten Präparaten herangezogen zu werden. In Hautschnitten lassen sich z. B. die Chromato. phoren bei Leucoderma syphiliticum auf diese Weise als hellleuchtende, gekörnte Zellen trefflich demonstrieren und studieren. Von Ringel- und Spindelha aren des Menschen, aber auch von den eigenartig beschaffenen, spindelförmigen Haaren des Maulwurfs und denen der Fledermaus gewinnt man im Kanadabalsamoder Oelpräparat sehr instruktive Bilder. (Kutikula, Pignient usw.). Auch Pilze, Kokken und Bakterien kann man als kleine, leuchtende Körperchen erkennen. Die Färbung der Kerne, die Struktur des Bindegewebes und anderes tritt in Schnittpräparaten oft schön leuchtend hervor, sodaß man farbenprächtige plastisclie, manchmal fast stereoskopische Bilder erhält. Als ich meinen Assistenten diese einfache Methode, die der Student schon beim a n a tomischen. Unterricht erlernen müßte, zuerst demonstrierte, waren sie von der Schönheit und Deutlichkeit der Bilder außerordentlich überrascht. Notwendig ist allerdings, da $B$ man mit der genannten Mattscheibe, die halb geölt, halb nicht geölt ist, stets für günstige Abblendung des Lichtes der Lampe sorgt. Wir sind jetzt damit beschäftigt, die Methode für die verschiedenen Gewebsstrukturen,

Aus der Universitäts-Hautklinik in Bonn.

\section{Ueber die Verwendung des Dunkelfeldes zur Auffindung der Gelbfieber-, Gelbsucht-, Syphilis- und anderer Spirochäten in fixierten und gefärbten Ausstrich - und Schnittpräparaten.}

\section{Von Erich Hoffmann. ${ }^{\mathbf{1}}$ )}

$\mathrm{H}$. Nog u chi ist es anscheinend gelungen, die Ursache des $\mathrm{Gelb}$ fiebers in einem von ihm als Leptospira icteroides benanten, feinen, der Spirochäte der Weilschen Krankheit ähnelnden Mikroorganismus aufzufinden 2). Er war so freundlich, mir nicht nur ein nach der Levaditi-Methode gefärbtes Schnittpräparat einer Niére, sondern auch Ausstriche von Blut, Niere und Leber eines mit den Gelbfieberspirochäten geimpften Meersclıweinchens zu übersenden, die reichlich Leptospiren enthalten. Die feinen, dem Typus der Weilschen oder Gelbsuchtspirochäte sowie meiner Spir. trimerodonta (Leptospira dentium) des Zahnbelags ${ }^{3}$ ) entsprechenden Spirochäten sind zum Teil nur schwach rötlich gefärbt, soda $\beta$ ihre Demonstration, besonders im Blutausstrich, Schwierigkeiten macht. Mit größter Leichtigkeit aber ließen sich diese feinen Mikroorganisment auch dem weniger geübten Mikroskopiker ohne weiteres demonstrieren, als icl diese gefärbten und fixierten Präparate mit dem ZeiBschen oder Leitzschen Dunkelfeld ${ }^{4}$ ) untersuchte. Hierbei erscheinen die Spirochäten, wenn mant das Licht der gebräuchlichen Liliputbogenlampe durch eine zur Hälfte geölte Mattscheibe etwas dämpft, grünlich fluoreszierend und treten sehr scharf hervor, lassen auch oft ihre feinen Windungent gut erkennen und die kleiderbügelähnlich abgebogenen Enden prächtig aufleuchten. Auch die Gelbsucht- und Syphilisspirochäte sowie andere Spirochätenformen sind auf diese Weise selbst in abgeblaßten Ausstrichen deutlich zu erkennen, wie ich an meinen ältesten, aus den Monaten März und April des Jahres 1905 stammenden Giemsa-Präparaten feststellen konnte ${ }^{5}$ ). All gröberen, mit der Löfflerschen Geißelfärbung dargestellten Balanitisspirochäten erscheint dabei der Körper gelbrötlich, während die spitz auslaufenden Endfäden mehr weiß aussehen. Aber auch in Schnittpräparaten, die nach der Levaditi-Methode gefärbt sind, lassen sich sowohl die Syphilisspirochäten, z. B im Gehirn und der Aorta von Paralytikern und in der Kaninchenkornea als auch die Spirochäten der Weilschen Krankheit und des Gelbfiebers ${ }^{6}$ ) in ihrer charakteristischen Form recht gut erkennen und leuchten bei richtiger Abblendung prächtig weiß auf. Auch sonst verdient die Dunkelfeldmethode zur Untersuchung von fixier-

1) Nach einem Vortrag in der Medizinischen Abteilung der Niederrheinischen Gesellschaft für Natur- und Heilkunde in Bonn am 13. XII. 1920. - ${ }^{2}$ ) Schon Fritz $S$ chaud inn hatte die Vermutung, daB eine Spirochăte die Ursache des Gellffiebers sei, und Stims on hat einmal in einer menschlichen Niere mittels der Levaditi-Methade spirochătenahntliche Gebilde bei Gerbfieber dargestellt (s. Kolle-Hets ch 25 . Auff. spirochătenas a) D. m. W. $1920 \mathrm{Nr} .10$ u. 23. - 4) Das erstere habe ich mehr benutzt, S. 1095 ). $-{ }^{-8}$ ) D. m. W. $1920 \mathrm{Nr} .10$ u. $23 .-{ }^{-4}$ ) Das erstere habe ich mehr benutzt,
besonders auch mit schwächeren Objektiven.
-5) Störend wirken in so alten Präbesonders auch mit schwächeren Objektiven. - -5) Störend wirken in so alten Präparaten zuweilen ausgefallene Kristalle; am besteit sind die mit $0 \mathrm{sm}$ iu m fixierten Ausstriche (nach der von $\mathrm{mir}$ und $\mathrm{Halle}$ angegebenen, Methode). - - $\left.{ }^{6}\right)$ Bezuglich der Penennung dieser netuen Spirochäten herrsclit - leider keine Einigkeit; Noguchi nennt das Gelbsuchtschräubchen Leptospira icterohaemorrhagiae, das Gelbfieberschräubchen Leptospira icteroides. Tch habe als. Gruppennamien wegen der Dreiteil ung Spirochaeta tri meres : orgeschlagen und henenne die Arten Spirochaeta icterohaemorrhagiae, Spirochaeta icteroides ind die im Mund vorkommende Spirochata trimerodonta. Die im Wasser von Uhle nhuth und $Z_{u}$ elzer beschriebene Form könte schlechthin als $S$ piroch aet a trimeres beZuelzer beschriebene Form könnte schlechthin. als Spirocha et a trimeres be-
zeichnet. Werden. (Vgl. D. m. W. 1920 Nr. 10 u. 23.) Auf die prinzipielle Frage der Berechtigung der Abtrennung der Gattung "Spirochaeta trimeres" oder "Leptospira ${ }^{4}$ (Form, Bewegungsart, Widerstand sältigkeit gegen 10\% ige Saponinlösung kann ich hier nicht eingehen und verweise auf die Arbeit meines Schifilers $\mathrm{Ed} \mathrm{mund}$ Hofmann ther Leptospira dentium. Zelleinlagerungen (Xanthomzellen usw.), Mikroorganismen und Färbuncell durchzuprüfen, und wollell auch den $Z$ e i $B$ sclien We chs el. k ond ens or für diese Untersuchungen heranziehen. Ich glaube aber, $\mathrm{da} B$ diese Methode sich nicht nur zar Auffindung spärlicher Sprrochäten, Pilze usw. in fixierten und gefärbten Präparaten und nicht nur zum Studium der Chromatophoren eignet, sondern auch sonst vesonders fürdie Demoustration beim Unterricht, Gutes leisten wird. Selbstverständ'ich fehlt hier die bei der Betrachtung lebender Bilder noch hinzukommende Bewegung, die für viele Mikroorganismen ja sehr kennzeichnend ist; aber auch ohne diese gibt das

Dunkelfeld so charakteristische und scharfe Bilder, daß es $\mathrm{zur} B \mathrm{Be}$ trachtung fixierter und gefärbter Präparate ange. legentlichst empfohlen werden kann.

Nachtrag. Nach Abschluß dieser Arbeit erhalte ich erst den Aufsatz des bewährten Spirochätenforschers Dr. Oelze (Leipzig) (Derm. Wschr. 71 Nr. 42), der den Zeißschen Wechselkon. de n s or benutzt und lehrreiche vergleichende Untersuchungen über die Ergiebigkeit der verschiedenen Methoden (Dunkelfeld, Negativdarstellung, Färbung) zur Auffindung voin Syphilisspirochäten angestellt hat. Er zeigt, daß nächst der Dunkelf elduntersuchung der frischen Präparate meine Osmium-Giemsa-Me tho d e ${ }^{1}$ die meisten Spirochäten (ca. $60-70 \%$ gegenüber $7-35 \%$ be andern Methoden) zur Anschauung bringt. Durch die von mir erprobte Dunkelfeldprüfung gefärbter Giemsa-Präparate, die auch er nicht erwähnt, dürfte man alle Spirochäten, selbst die auf und unter Zellen liegenden, auffinden können. Die Endfäden und die Endkörnchen der Gelbfieber-, Gelbsuchtund anderer Spirochäten sind auf diese Weise trefflich zu demonstrieren; auch in Schnitten sieht man schlecht versilberte Exemplare gut und erhält dadurch eine bessere Ausbeute. Her Prof Schmidt, der während des Krieges in meinem Laboratorium gearbeitet hat hat schon damals die Vorzüge des Dúnkelfelds für das Studium der Chromatophoren bei [Reptilien ${ }^{2}$ ) kennen gelernt und das in seinen Arbeiten erwähnt.

Eine genatiere Darstellung der Leuch tbildmethode und ihrer Bedeutung für die Auffindung von Tuberkelbazillen usw. folgt alsbald in der B. kl. W. (nach einem am 5. 1. 1921 in der Berliner medizinischen Gesellschaft gehaltenem Vortrage).

1) Vgl. E. H of tma n n, Behandlung der Haut- und Geschlechtskrankheiten. 3. Aufl. S. 127. Dem Aurstrich vorausgehende Osmierung der Objektträger ist empfehlenswert (vg1. meine Aetiologie der Syphilis). - 2) W. J. Schmidt, Die Chromatophoren der Reptilienhaut, Arch. f. mikr. Anat. $90 \mathrm{Abt}$. I $1917 \mathrm{~S}$. 201. (Wir wenden jetzt den Paraboloidkondensor, dazu Obj. $X$ und die Komp. Ok. 4 und 12 an.) - 